\title{
Muscular Hydatidosis Mimicking Lipoma
}

MARIA COLAZO-BURLATO, MD, Department of Rheumatology, Hospital Universitario de Burgos; BONIFACIO ALVAREZ-LARIO, MD, PhD, Department of Rheumatology, Hospital Universitario de Burgos, Burgos, Spain. Address correspondence to Dr. M. Colazo-Burlato, Department of Rheumatology, Hospital Universitario de Burgos, Islas Baleares, Av 3, 09006, Burgos, Spain. E-mail: maria_colazo@hotmail.com. The patient's consent could not be obtained because the followup was lost. An assessment of the case was requested from the Burgos Hospital Ethics Committee, which found no objection to its publication (Ref. No. CEIC 1855). J Rheumatol 2018;45:1602; doi:10.3899/jrheum.171126

Echinococcal cysts are usually found in liver and lungs (90\%); spleen, heart, and brain are affected more rarely. Muscular hydatidosis accounts for $<1 \%$ of all cases, the most common locations being the thigh and paravertebral muscles ${ }^{1,2}$.

A 51-year-old Spanish male with a painless mass in his left thigh was referred to the rheumatology clinic in the University Hospital of Burgos in 2014, and the mass was diagnosed as probable lipoma. He was living in a small city and had contact with hunting dogs. Ultrasound examination of the mass showed a multilocular cyst. Magnetic resonance imaging (MRI) revealed a well-demarcated $12 \times 6 \times 5$-cm cystic lesion located in the adductor magnus muscle with an outline delineating it from the surrounding muscles (Figure 1). Inside the mother cyst were multiple smaller ("daughter") cysts ${ }^{3}$. Serology for Echinococcus by ELISA was negative at the time of diagnosis; however, the patient was studied 25 years earlier because of positive serology for hydatid disease, with normal abdominal ultrasonography, chest radiograph, and brain and bone scanning. Diagnosis of muscular echinococcosis was made, and he was treated surgically.
Histopathological diagnosis confirmed the diagnosis of hydatid disease. No recurrence was seen in 2 years.

Serology for hyatid disease may be false-negative in senescent or dead cysts and in extrahepatic sites such as the skeletal muscle. The treatment of choice is surgery, with the adjuvant use of albendazole to prevent dissemination ${ }^{4}$.

Muscular hydatidosis should be considered for every soft cystic mass in any anatomical location, in individuals who live in or have immigrated from endemic areas.

\section{REFERENCES}

1. McManus DP, Zhang W, Li J, Bartley PB. Echinococcosis. Lancet 2003;362:1295-304.

2. Tekin R, Avci A, Tekin RC, Gem M, Cevik R. Hydatid cysts in muscles: clinical manifestations, diagnosis, and management of this atypical presentation. Rev Soc Bras Med Trop 2015;48:594-8.

3. Garcia-Diez AI, Ros Mendoza LH, Villacampa VM, Cozar M, Fuertes MI. MRI evaluation of soft tissue hydatid disease. Eur Radiol 2000;10:462-6.

4. Thursky K, Torresi J. Primary muscle hydatidosis of the thigh: Management of a complicated case with combination adjunctive albendazole and praziquantel chemotherapy. Clin Infect Dis 2001;32:e65-8.

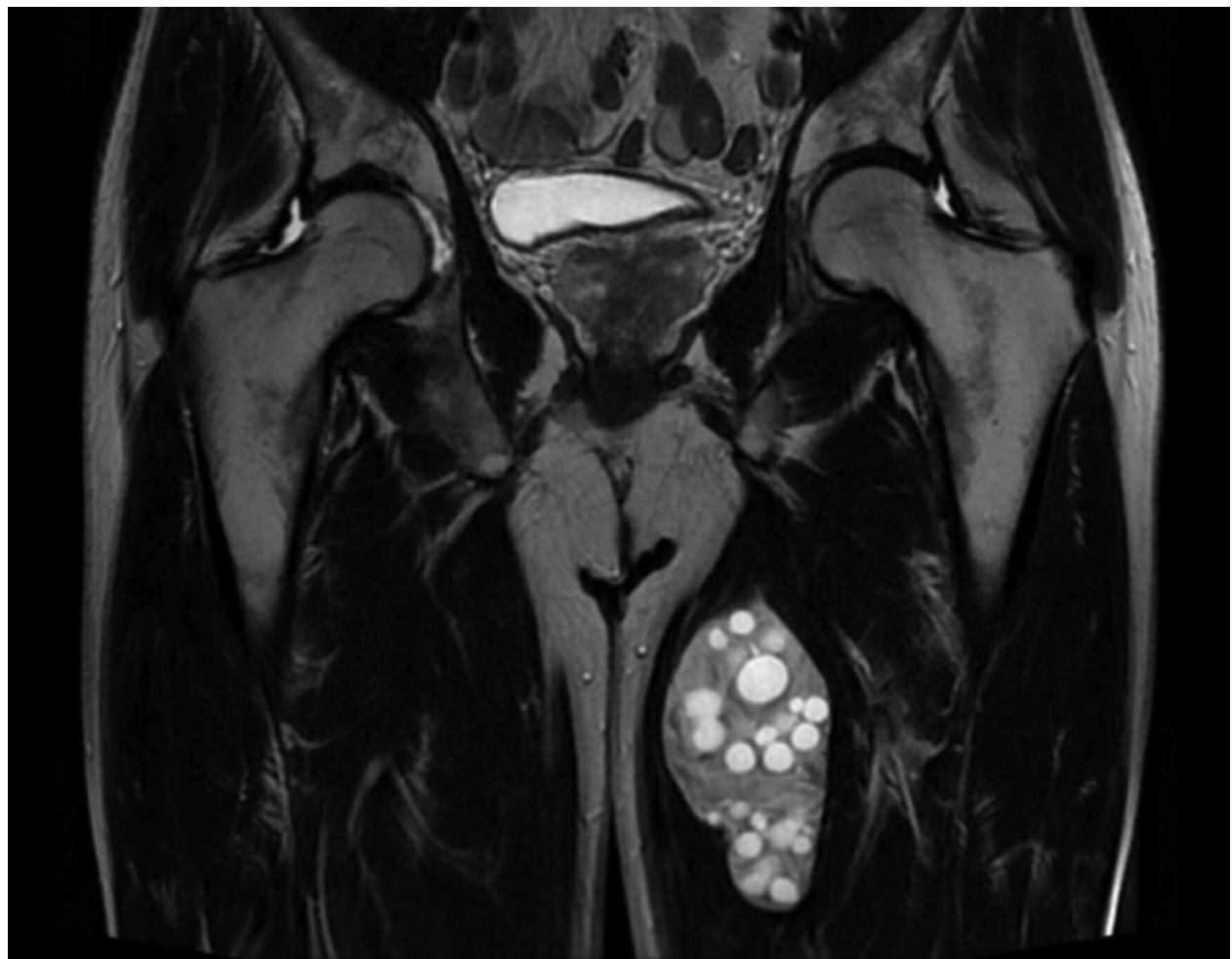

Figure 1. Coronal T2-weighted MRI of a cystic mass arising from the left adductor magnus muscle. A hyperintense well-defined cyst containing multiple daughter cysts is seen. MRI: magnetic resonance imaging.

Personal non-commercial use only. The Journal of Rheumatology Copyright @ 2018 . All rights reserved. 